\title{
Measurement-Induced Phase Transition in the Monitored Sachdev-Ye-Kitaev Model
}

\author{
Shao-Kai Jian $\odot,{ }^{1, *}$ Chunxiao Liu $\odot,{ }^{2, *}$ Xiao Chen, ${ }^{3, \dagger}$ Brian Swingle, ${ }^{4,1, \ddagger}$ and Pengfei Zhang ${ }^{5, \S}$ \\ ${ }^{1}$ Condensed Matter Theory Center and Joint Quantum Institute, Department of Physics, \\ University of Maryland, College Park, Maryland 20742, USA \\ ${ }^{2}$ Department of Physics, University of California Santa Barbara, Santa Barbara, California 93106, USA \\ ${ }^{3}$ Department of Physics, Boston College, Chestnut Hill, Massachusetts 02467, USA \\ ${ }^{4}$ Department of Physics, Brandeis University, Waltham, Massachusetts 02453, USA \\ ${ }^{5}$ Institute for Quantum Information and Matter and Walter Burke Institute for Theoretical Physics, \\ California Institute of Technology, Pasadena, California 91125, USA
}

(Received 28 April 2021; revised 30 July 2021; accepted 17 August 2021; published 27 September 2021)

\begin{abstract}
We construct Brownian Sachdev-Ye-Kitaev (SYK) chains subjected to continuous monitoring and explore possible entanglement phase transitions therein. We analytically derive the effective action in the large- $N$ limit and show that an entanglement transition is caused by the symmetry breaking in the enlarged replica space. In the noninteracting case with $\mathrm{SYK}_{2}$ chains, the model features a continuous $O(2)$ symmetry between two replicas and a transition corresponding to spontaneous breaking of that symmetry upon varying the measurement rate. In the symmetry broken phase at low measurement rate, the emergent replica criticality associated with the Goldstone mode leads to a log-scaling entanglement entropy that can be attributed to the free energy of vortices. In the symmetric phase at higher measurement rate, the entanglement entropy obeys area-law scaling. In the interacting case, the continuous $O(2)$ symmetry is explicitly lowered to a discrete $C_{4}$ symmetry, giving rise to volume-law entanglement entropy in the symmetry-broken phase due to the enhanced linear free energy cost of domain walls compared to vortices. The interacting transition is described by $C_{4}$ symmetry breaking. We also verify the large- $N$ critical exponents by numerically solving the Schwinger-Dyson equation.
\end{abstract}

DOI: 10.1103/PhysRevLett.127.140601

Introduction.-Quantum dynamics can be nonunitary provided that the process occurs with a probability less than one, with the central example being measurement. Fathoming the effects of nonunitary evolution on manybody quantum states has emerged as a frontier in recent years, although the issues at play touch on the foundations of quantum physics. In particular, nonunitary evolution can lead to dramatic phase transitions in the entanglement structure of a many-body state, as in the recently discovered paradigm of the measurement-induced phase transition realized in local random unitary circuits interspersed with measurements [1-5], in which the steady-state entanglement entropy changes from volume-law scaling to area-law scaling upon increasing the measurement rate.

This transition has been observed in various settings including random Haar circuits, random Clifford circuits, Floquet quantum circuits, etc., [6-14]. It is continuous and enjoys an emergent conformal symmetry at the critical point. Entanglement transitions were also studied in the context of $\mathcal{P} \mathcal{T}$ symmetry breaking, where the physics mechanism might be different [15-20]. Moreover, this transition finds important applications in revealing phase transitions in quantum error correcting codes [4,21,22], proving efficiency in classical simulations of random shallow quantum circuits [23], and so on. In addition to measurement-induced phase transitions in random hybrid circuits, an interesting distinct class of phenomena arise in noninteracting fermion circuits, which can host a critical phase when subjected to weak measurements. In this situation, the entanglement entropy shows a log scaling with subsystem size in an entire phase instead of just at the transition point [24-29]. As the measurement strength is further increased, a phase transition from the critical phase to the area-law phase occurs $[25,29]$.

The phases and transitions arising from nonunitary dynamics are typically only visible in entropic observables which are nonlinear in the density matrix. Accessing entropic observables averaged over the various sources of randomness thus requires averaging multiple replicas of the system with identical randomness. Depending on the observable of interest, one then has to take various kinds of replica limits. In the random Haar hybrid circuit, it is argued that the entanglement transition problem can be mapped to an order-disorder transition of a complicated statistical mechanical model in the replicated space [3, 30-32]. In the free fermion model, the critical phase is accounted for by a Goldstone mode resulting from the spontaneous breaking of a continuous replica rotational symmetry $[28,29,33]$. 


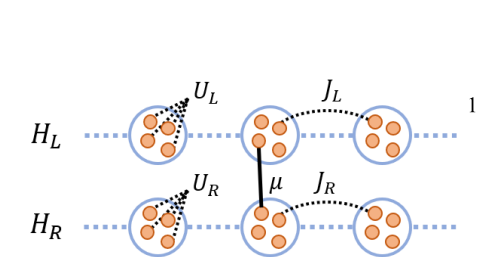

(a)

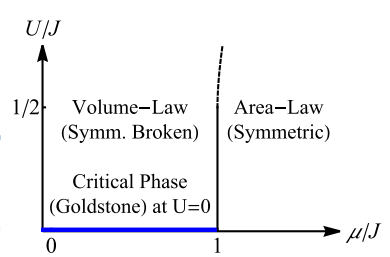

(b)

FIG. 1. (a) A schematic plot of the monitored SYK chains. The dashed (solid) line represents Brownian random couplings (monitoring operators). (b) The phase diagram of the model at infinite $N$. The black solid (dashed) line at $\mu=J$ denotes the continuous (discontinuous) transition. The blue thick line denotes the critical phase for the noninteracting case.

In light of these developments, it is of great interest to construct a solvable model in which these measurementdriven transitions and critical phases can be analytically understood systematically. With this motivation in mind, we consider Brownian Sachdev-Kitaev-Ye (SYK) chains [34-40] subjected to continuous monitoring, and explore possible phases and transitions. Previously, the SYK model has been extensively used to analytically understand quantum chaos and quantum information dynamics [41-48]. We find that varying the monitoring rate in the nonunitary SYK dynamics causes a measurementinduced phase transition corresponding to the spontaneous breaking of $C_{4}$ or $O(2)$ symmetry depending on whether the model is interacting or not (see Fig. 1). We extract the effective action describing the symmetry breaking and evaluate the subsystem entanglement entropy by mapping to the free energies of topological defects created by the twisted boundary conditions. We further obtain various critical exponents at the critical point and in the critical phase.

Model and setup.-Consider the following Brownian Hamiltonian describing left $(L)$ and right $(R)$ chains with $\mathrm{SYK}_{2}$ hoppings and $\mathrm{SYK}_{q}$ on-site interactions [49,50],

$$
\begin{aligned}
H= & \sum_{x ; a=L, R}\left[\sum_{i j} i J_{a, i j}^{x, x+1}(t) \psi_{x, a, i} \psi_{x+1, a, j}\right. \\
& \left.+\sum_{j_{1}<\cdots<j_{q}} i^{q / 2} U_{a, j_{1} \ldots j_{q}}^{x}(t) \psi_{x, a, j_{1}} \ldots \psi_{x, a, j_{q}}\right],
\end{aligned}
$$

where $\psi_{x, a, i} i=1, \ldots, N$ denotes $i$ th of $N$ Majorana fermion at each site $x=1, \ldots, L$ of the $a=L, R$ chains. $L$ is the number of sites and periodic boundary conditions are assumed in this Letter. In the second line, $q \geq 4$ is an even integer indicating $q$-body interaction [36]. $J_{a, i j}^{x, x+1}$ $\left(U_{a, j_{1}, \ldots, j_{q}}^{x}\right)$ is the hopping (interaction) strength. The couplings in the left and right chains are independent Gaussian variables with mean zero and variances

$$
\begin{aligned}
\overline{J_{a, i j}^{x, x+1}\left(t_{1}\right) J_{a^{\prime}, i j}^{x^{\prime}, x^{\prime}+1}\left(t_{2}\right)} & =\frac{J_{a}}{2 N} \delta\left(t_{12}\right) \delta_{a a^{\prime}} \delta^{x, x^{\prime}}, \\
\overline{U_{a, j_{1} \ldots j_{q}}^{x}\left(t_{1}\right) U_{a^{\prime}, j_{1} \ldots j_{q}}^{x^{\prime}}\left(t_{2}\right)} & =\frac{2^{q-2}(q-1) ! U_{a}}{N^{q-1}} \delta\left(t_{12}\right) \delta_{a a^{\prime}} \delta^{x, x^{\prime}} .
\end{aligned}
$$

The time dependence and Dirac $\delta$ functions indicate the Brownian nature of the couplings. For simplicity, we set $J_{L}=J_{R}=J$ and $U_{L}=U_{R}=U$ throughout the Letter.

In addition, the system is under continuous monitoring: in each infinitesimal time step $\delta t$, we apply a measurement with probability $p$ at every site. The local measurement operator couples the $L$ and $R$ fermions at each site, as described by the operators

$$
\left\{M_{1}^{x, i}, M_{2}^{x, i}\right\}=\left\{\pi_{x, i}^{-}+\sqrt{1-s^{2}} \pi_{x, i}^{+}, s \pi_{x, i}^{+}\right\},
$$

where $\pi_{x, i}^{ \pm}=\frac{1}{2}\left(1 \mp i 2 \psi_{x, L, i} \psi_{x, R, i}\right)$ is the projection to one of the Fermi parity eigenstates and $0<s \leq 1$ is the measurement strength. Notice that $M_{1}^{x, i}$ and $M_{2}^{x, i}$ satisfy the required completeness relation $M_{1}^{x, i \dagger} M_{1}^{x, i}+M_{2}^{x, i \dagger} M_{2}^{x, i}=I$.

It is convenient to further introduce the Kraus operator, $K_{\mu}^{x, i}=\left\{I, M_{1}^{x, i}, M_{2}^{x, i}\right\}$ with weights $w_{0}=(1-p), w_{1}=$ $w_{2}=p$ [26]. During each time step in $\delta t$, the evolution of the non-normalized density matrix for a quantum trajectory with measurement outcome $\mu_{x, i}$ is [51-54]

$$
\begin{aligned}
& \rho_{a b}\left(\delta t ; \mu_{x, i}\right) \\
& =\left(\bigotimes_{x, i} K_{\mu_{x, i}}^{x, i}\right)_{a c}\left(\bigotimes_{x, i} K_{\mu_{x, i}}^{x, i \dagger}\right)_{d b}\left(e^{-i H \delta t}\right)_{c e}\left(e^{i H \delta t}\right)_{f d} \rho_{e f} .
\end{aligned}
$$

For observables linear in density matrix, one can perform the average in $\rho$. If measurement of a specific $i$ th Majorana at site $x$ is performed, the average change of density matrix (we suppress index $x, i)$, is $\sum_{\nu}\left(w_{\nu} K_{\nu} \rho K_{\nu}^{\dagger}\right)-\rho \approx$ $-\left(p s^{2} / 2\right)\left\{\pi^{+}, \rho\right\}+p s^{2} \pi^{+} \rho \pi^{+}$, where we keep up to $O\left(s^{2}\right)$ order. Let $s=(\mu / p) \sqrt{\delta t}$, we get the Lindblad equation $(d \rho / d t)=-\frac{1}{2}\left\{L^{\dagger} L, \rho\right\}+L \rho L^{\dagger}$, where $L=\mu \pi^{+}$ is the jump operator. For observables nonlinear in density matrix, one is not able to get a linear Lindblad equation. Therefore, we use the path integral formalism detailed below.

For our purpose, we are interested in calculating the quasi- $n$ entropy of bipartite system $A \bar{A}$ [23],

$$
S_{A}^{(n)}=\frac{1}{1-n} \log \frac{\mathbb{E} \operatorname{Tr}\left(\rho_{A}^{n}\right)}{\mathbb{E} \operatorname{Tr}(\rho)^{n}},
$$

where $\rho\left(\rho_{A}\right)$ is the total density matrix (the reduced density matrix of subsystem $A$ by tracing out $\bar{A}$ ), and $\mathbb{E}$ denotes the average over the Brownian variables and the continuous monitoring overcomes. To evaluate this quantity, one should generalize to $n$ replicas. We mainly consider $n=2$, and use a $1,2,3,4$ notation: $1,2(3,4)$ denote the 


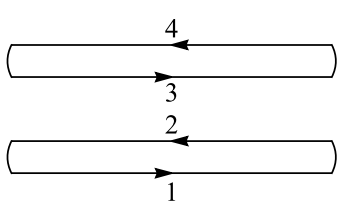

(a)

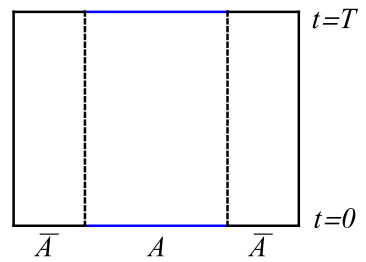

(c) $T \ll L_{A}$

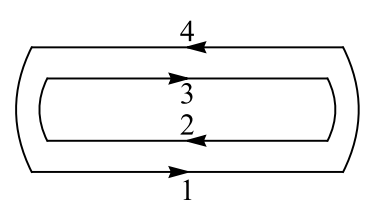

(b)

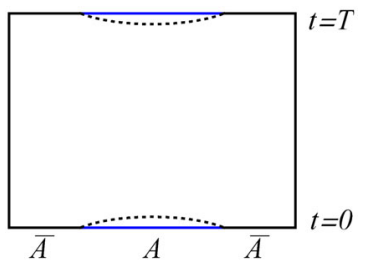

(d) $T \gg L_{A}$
FIG. 2. (a) The boundary condition corresponding to $\operatorname{Tr}(\rho)^{2}$. (b) The boundary condition corresponding to two twist operators inserted at $t=0$ and $t=T$, respectively. (c),(d) The twisted boundary conditions are indicated by the blue line in subsystem $A$. In the volume-law space, the spacetime domain wall indicated by the dashed curve separates two domains induced by different boundaries. Schematic plots of domain walls are shown at short times (c) $T \ll L_{A}$ and long times (d) $T \gg L_{A}$.

first (second) replica, and 1,3 $(2,4)$ denote the forward (backward) evolution. A schematic plot is given in Figs. 2(a) and 2(b). We use superscript Greek alphabet $\alpha=1,2,3,4$ to denote the contour.

To evaluate $\mathbb{E} \operatorname{Tr}(\rho)^{2}$, we derive the effective action governing the time evolution in the replicated space. The continuous monitoring at each step can be cast into

$\sum_{\nu} w_{\nu}\left(K_{\nu}^{x, i}\right)^{\otimes 2} \otimes\left(K_{\nu}^{x, i \dagger}\right)^{\otimes 2} \approx e^{(\mu \delta t / 2) \sum_{\alpha=1}^{4} i \psi_{x, L, i}^{\alpha} \psi_{x, R, i}^{\alpha},}$

which is obtained in the limit $p \ll s \ll 1[55] . \mu \equiv p s^{2} / \delta t$ is the relevant measurement rate that is kept fixed when the limit is taken. Then the effect of monitoring every Majorana species $i$ at every site $x$ is described by

$$
\exp \left(\frac{\mu}{2} \int d t \sum_{x, \alpha, i} i \psi_{x, L, i}^{\alpha} \psi_{x, R, i}^{\alpha}\right)
$$

where we implicitly sum over all infinitesimal time steps to arrive at the time integral for a time evolution.

Combining the Brownian Hamiltonian (1) and the measurement (7) and integrating out the Gaussian variables, the effective action governing the time evolution in the replicated space reads

$$
\begin{aligned}
-\frac{I}{N}= & \frac{1}{2} \operatorname{Tr} \log \left[(-1)^{\alpha+1} \partial_{t}-\Sigma_{x}\right)-\frac{1}{2} \int \Sigma_{a b, x}^{\alpha \beta} G_{a b, x}^{\alpha \beta} \\
& +\int \delta\left(t-t^{\prime}\right)\left\{-\frac{(-1)^{\alpha+\beta}}{4} \delta_{a b}\left[J G_{a b, x}^{\alpha \beta} G_{a b, x+1}^{\alpha \beta}\right.\right. \\
& \left.\left.+\frac{U}{2 q}\left(2 G_{a b, x}^{\alpha \beta}\right)^{q}\right]+\frac{i \mu}{2} G_{L R, x}^{\alpha \alpha}\right\},
\end{aligned}
$$

where $\alpha, \beta=1, \ldots, 4$ denote the four contours. The summations over $x, a, b$ and $\alpha, \beta$ are implicit. $\Sigma_{a b, x}^{\alpha \beta}\left(t, t^{\prime}\right)$ is the self-energy introduced to enforce $G_{a b, x}^{\alpha \beta}\left(t, t^{\prime}\right)=$ $(1 / N) \sum_{j} \psi_{x, a, j}^{\alpha}(t) \psi_{x, b, j}^{\beta}\left(t^{\prime}\right)$. Saddle-point analysis can be straightforwardly applied to the large- $N$ action [55].

Monitored $\mathrm{SYK}_{2}$ chain and $O(2)$ transition.-For the noninteracting case, $U=0$, the replica diagonal spatially uniform solution (with site index suppressed) reads

$\bar{G}= \begin{cases}\frac{e^{\frac{-J|t|}{2}}}{2}\left[\operatorname{sgn}(t) \sigma^{z}-\sqrt{1-\tilde{\mu}^{2}} i \sigma^{y}+\tilde{\mu} \tau^{y}\right], & \tilde{\mu}<1 \\ \frac{e^{\frac{\mu|t| t}{2}}}{2}\left[\operatorname{sgn}(t) \sigma^{z}+\tau^{y}\right], & \tilde{\mu} \geq 1,\end{cases}$

where $t$ is the time difference, $\tilde{\mu} \equiv \mu / J$ and Pauli matrix $\sigma$ $(\tau)$ acts on 1 and 2 contours ( $L$ and $R$ chains). The solution on 3,4 contours is the same, consistent with the boundary condition without twist operators [Fig. 2(a)].

First we look at the theory from a symmetry perspective. For Brownian randomness (2), it is legitimate to assume the Green functions are strictly local and antisymmetric $G_{a b, x}^{\alpha \beta}(t)=-G_{b a, x}^{\beta \alpha}(t)$ [37], so for $U=0$ the action becomes

$$
\begin{aligned}
-\frac{I}{N}= & \frac{1}{2} \operatorname{Tr} \log \left(S \partial_{t}+\Sigma_{x}\right) \\
& +\int \frac{1}{2} \operatorname{Tr}\left[\Sigma_{a b, x} G_{b a, x}+\frac{J}{4} G_{a b, x} S G_{b a, x+1} S+i \frac{\mu}{2} G_{L R, x}\right],
\end{aligned}
$$

where $S^{\alpha \beta}=(-1)^{\alpha} \delta^{\alpha \beta}$ and the trace in the second line is over the contours. For a finite measurement rate $\mu>0$, the theory features $O(2) \times O(2)$ symmetry [56],

$G_{a b, x} \rightarrow O^{-1} G_{a b, x} O, \quad O^{T} O=1, \quad O^{T} S O=S$,

where $O$ acts identically on the left and right chains [57]. The rotational symmetry is generated by $\gamma_{(13)}$ and $\gamma_{(24)}$, which is defined in component $\alpha, \beta$ by $\gamma_{(i j)}^{\alpha \beta}=$ $\delta^{i \alpha} \delta^{j \beta}-\delta^{j \alpha} \delta^{i \beta}$, acting on the replica space. Intuitively, one of the $O(2)$ symmetries is to rotate between 1 and 3 contours and the other to rotate between 2 and 4 contours.

The saddle-point solution (9) for $\mu<J$ spontaneously breaks the relative rotational symmetry, so there is one Goldstone mode, which is generated by applying the broken-symmetry generator $\gamma_{-} \equiv \gamma_{(13)}-\gamma_{(24)}$, i.e.,

$$
\begin{aligned}
\delta G_{a a, x}(t) & =e^{-\theta_{x}(t) \gamma_{-}} \bar{G}_{a a}(0) e^{\theta_{x}(t) \gamma_{-}}-\bar{G}_{a a}(0) \\
& \approx \sqrt{1-\tilde{\mu}^{2}} \theta_{x}(t)\left(\gamma_{(14)}+\gamma_{(23)}\right)
\end{aligned}
$$

where $\theta_{x}(t)$ denotes the Goldstone mode, and in the second line we assume the fluctuation is small, $\theta_{x}(t) \ll 1$. We anticipate that it will dominate at low energies at $\mu<J$. 
In contrast, when $\mu>J$, this $O(2)$ symmetry is unbroken and the replicated theory is in the gapped phase.

With this understanding, we are ready to evaluate the effective action for the Goldstone mode. First notice that $G_{L R}^{\alpha \alpha}$ is linear in the action (10), so it can be integrated out to enforce $\Sigma_{L R}^{\alpha \alpha}=(i \mu / 2)$. Then we consider the fluctuations $\delta \Sigma_{a a}^{\alpha \beta}$ and $\delta G_{a a}^{\alpha \beta}$ away from the saddle-point solution (9) at $\mu<J$. The effective theory for the Goldstone mode reads [55],

$$
\frac{I_{\mathrm{eff}}}{N}=\frac{\rho}{2} \sum_{k} \int_{\Omega}\left(\frac{\Omega^{2}}{\mu^{2}}+(1-\cos k)\right)\left|\theta_{k}(\Omega)\right|^{2},
$$

where $\int_{\Omega}=\int(d \Omega / 2 \pi)$ and $\theta_{k}=(1 / \sqrt{L}) \sum_{x} \theta_{x} e^{-i k x}$ is the Fourier transform of the lattice site. The stiffness $\rho=$ $J\left(1-\tilde{\mu}^{2}\right)$ vanishes at $\tilde{\mu}=1$, indicating that the transition occurs at $\mu=J$, which is expected because the saddlepoint solution restores $O(2)$ symmetry. Recently, a similar mechanism was discussed in the small- $N$ case in the language of Kosterlitz-Thouless transition in Refs. [28,29]. The Goldstone mode also explains the power-law squared correlation function of fermions in the critical phase $[24,25,33,55]$.

Interacting $\mathrm{SYK}_{4}$ model and $C_{4}$ transition.-In the interacting case $U>0$, when $\mu<J$, the uniform solution reads

$\bar{G}=\frac{e^{-\left(J+U \zeta^{q-2} / 2\right)|t|}}{2}\left[\operatorname{sgn}(t) \sigma^{z}-\zeta i \sigma^{y}+\frac{\tilde{\mu} \tau^{y}}{1+\tilde{U} \zeta^{q-2}}\right]$,

with $\quad \tilde{U} \equiv U / J$. The parameter $\zeta$ is given by $\left(1-\zeta^{2}\right)\left(1+\tilde{U} \zeta^{q-2}\right)^{2}=\tilde{\mu}^{2} . \zeta$ characterizes the correlation between forward and backward contours, and serves as an order parameter as we will see later. For small $\tilde{U}, \zeta=$ $\sqrt{1-\tilde{\mu}^{2}}\left[1+\tilde{\mu}^{2}\left(1-\tilde{\mu}^{2}\right)^{q / 2-2} \tilde{U}+O\left(\tilde{U}^{2}\right)\right]$ is well defined when $\mu<J$, and vanishes continuous as $\mu \rightarrow J$. In the following we will focus on the simplest interacting case with $q=4$, while our results are true for general $q$. At the critical point,

$$
\zeta^{2}\left[(2 \tilde{U}-1)+\left(\tilde{U}^{2}-2 \tilde{U}\right) \zeta^{2}-\tilde{U}^{2} \zeta^{4}\right]=0,
$$

which shows that for $\tilde{U}>1 / 2$ there are two degenerate distinct physical solutions indicating a discontinuous jump. Thus, the condition for a continuous transition is $2 U<J$. On the other hand, when $\mu \geq J$, the solution is the same as the noninteracting case (9) at $\tilde{\mu} \geq 1$.

For $U>0$ apparently the action cannot be cast into such a nice form as (10), so what symmetry out of $O(2) \times O(2)$ is preserved? It is easy to show that the symmetry reduces to $C_{4} \times C_{4}$, satisfying the condition $\left[\left(O^{-1}\right)^{\alpha \beta}\right]^{q / 2} S^{\beta \gamma}\left(O^{\gamma \delta}\right)^{q / 2}=S^{\alpha \delta}$. The generator is still given by $\gamma_{(13)}$ and $\gamma_{(24)}$ but the rotation angle is restricted to multiples of $\pi / 2$. The relative rotation symmetry is spontaneously broken by nonzero $\zeta$ in (15) when $\mu<J$. Namely, $\zeta$ serves as an order parameter of the $C_{4}$ symmetry breaking transition.

Aiming at the critical theory, we consider the effective theory of fluctuations from the symmetric saddle-point solution [55],

$$
\begin{aligned}
\frac{I_{\mathrm{eff}}}{N}= & \frac{1}{2} \sum_{i=1,2 ; k} \int_{\Omega}\left[\frac{\Omega^{2}}{\mu}+J(1-\cos k)\right]\left|\phi_{i, k}(\Omega)\right|^{2} \\
& +\sum_{x} \int_{t}\left[\frac{\mu-J}{2} \vec{\phi}_{x}^{2}+\frac{\mu}{8} \vec{\phi}_{x}^{4}-\frac{U}{4}\left(\phi_{1, x}^{4}+\phi_{2, x}^{4}\right)\right],
\end{aligned}
$$

where $\int_{t}=\int d t, \phi_{1}=\delta G^{12}+\delta G^{34}$, and $\phi_{2}=\delta G^{14}+\delta G^{23}$ [58] transform like a vector under the relative $C_{4}$ rotation. This theory features a second order transition if $2 U<\mu$, and a first order one if $2 U>\mu$, consistent with the analysis (16) of the saddle-point solution.

Entanglement transition and spacetime domain wall.After analyzing the effective action for the averaged $\mathbb{E} \operatorname{Tr}(\rho)^{2}$, we are now ready to study the entanglement transition. Importantly, the quasi- $n$ entropy (5) tends to trajectory-averaged entanglement entropy at $n \rightarrow 1$ limit just like the Rényi- $n$ entropy, which gives some justification of using quasi-2 entropy in the following as a proxy of entanglement entropy [59]. To observe the entanglement transition, we start from the well-known thermofield double state (TFD) in the doubled Hilbert space $[42,47,60]$. The entropy of subsystem $A$ at time $T / 2$ is obtained by imposing two twist operators at time $t=0$ and $t=T$ in the subsystem $A$ which change the boundary condition by requiring $G_{a a}^{14}(0)=G_{a a}^{23}(0)=G_{a a}^{14}(T)=G_{a a}^{23}(T)=\frac{1}{2}$ as indicated in Fig. 2(b). Equivalently in terms of the $Z_{4}$ model (17) of the interacting case, the boundary of $A$ has $\left(\phi_{1}=0, \phi_{2}>0\right)$ whereas that of $\bar{A}$ has $\left(\phi_{1}>0, \phi_{2}=0\right)$. In the symmetry-broken phase, it amounts to create distinct space time domains and consequently domain walls separating them as indicated in Figs. 2(c) or 2(d). Then the quasientropy is given by the free energy difference between the configurations with and without twisted boundary conditions.

Redefining $Z_{4}$ the theory (17) to be

$\frac{I_{\mathrm{eff}}}{N}=\int d t d x\left[\frac{1}{2}(\partial \vec{\phi})^{2}+r \vec{\phi}^{2}+\lambda \vec{\phi}^{4}+\lambda^{\prime}\left(\phi_{1}^{4}+\phi_{2}^{4}\right)\right]$,

with $r=\frac{1}{2} \mu(\mu-J), \lambda=(\sqrt{2} / 8) \mu^{\frac{5}{2}} J^{-\frac{1}{2}}$, and $\lambda^{\prime}=-(2 U / \mu) \lambda$, one can find that the surface tension of domain walls reads

$$
\sigma=\frac{\pi J \tilde{U}^{\frac{1}{2}}}{2 \sqrt{2}}\left(\frac{1-\tilde{\mu}}{\tilde{\mu}-2 \tilde{U}}\right)^{3 / 2} .
$$

This can also be estimated as $\sigma \sim \xi\left(\phi_{0}^{2} / \xi^{2}\right) \sim r^{3 / 2}$, where $\phi_{0}=|\langle\vec{\phi}\rangle| \sim \sqrt{r}$ and the correlation length $\xi \sim 1 / \sqrt{r}$. 


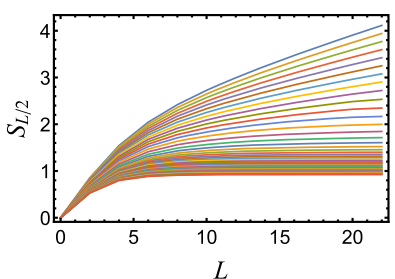

(a)

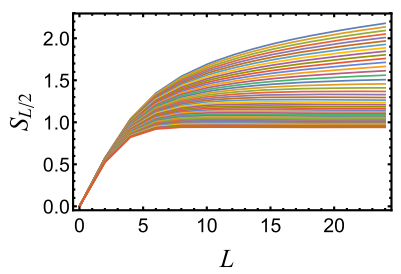

(c)

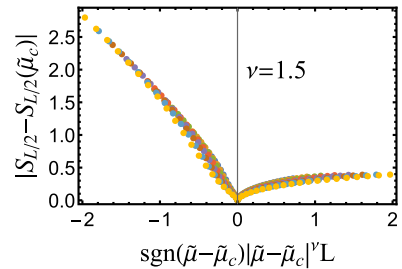

(b)

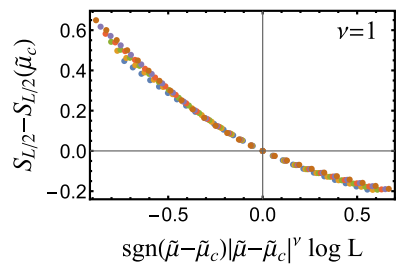

(d)
FIG. 3. The half-chain quasientropy as a function of the length of the chain. Different curves represent different measurement strength $\tilde{\mu}=0.8,0.81, \ldots, 1.2$ for (a) $U=0.4 \mathrm{~J}$ and (c) $U=0$. Data collapse verifies the critical exponent for (b) $U=0.4 J$ and (d) $U=0$.

With this information, the quasientropy in the volume-law phase is given by

$$
S_{A}^{(2)}= \begin{cases}2(N \sigma T+\log T / 2), & T \ll L_{A} \\ 2\left(N \sigma L_{A}+3 / 2 \log L_{A}\right), & T \gg L_{A},\end{cases}
$$

with $L_{A}$ the length of subsystem $A$. The factor of 2 is due to the doubling of the Hilbert space in defining the TFD initial state. The leading order term is intuitively shown by the configurations in Figs. 2(c) and 2(d), while the subleading $1 / N$ logarithmic correction is from the transverse fluctuations. Indeed this is the capillary wave theory of the volume-law phase discussed in Ref. [22]. Approaching the critical point $\tilde{\mu} \rightarrow 1$, the surface tension (19) vanishes continuously with an exponent $\nu=3 / 2$, and consequently the system undergoes an entanglement phase transition. The critical exponent is verified numerically by solving the Schwinger-Dyson equation [55] as shown in Figs. 3(a), 3 (b). Furthermore, the purification transition can be understood in a similar way [61].

This geometric picture is a systematic way to understand the connection between the volume-scaling and the log-scaling entanglement entropy in the interacting and noninteracting cases respectively: the surface tension (19) vanishes when the interaction strength is zero, and the domain walls created by the twisted boundary conditions in $\phi$ field change to the vortices in $\theta$ field [33]. A similar calculation shows at the leading order $S_{A}^{(2)} \sim$ $(1-\tilde{\mu}) N \log L_{A}$, that is well expected from the logarithmic free energy of vortices. The critical exponent $\nu=1$ is verified numerically in Figs. 3(c), 3(d). In addition, we

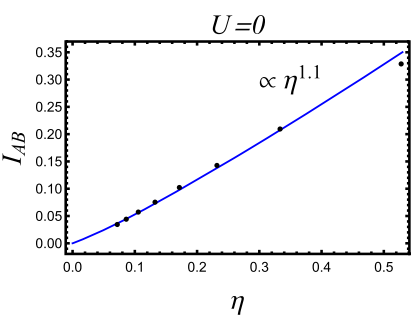

(a)

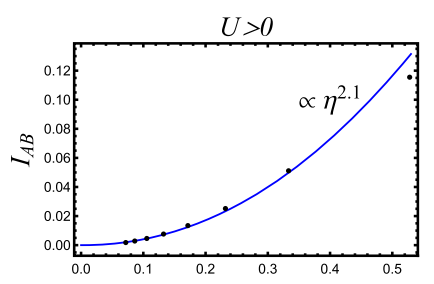

$\eta$
FIG. 4. Mutual information as a function of cross ratio $\eta$. The mutual information $I_{A B}$ is between $A$ and $B$, two symmetric intervals $L_{A}=L_{B}=2$ at opposite sides in the chain of length $L=10, \ldots, 24$. (a) The free case with $\tilde{\mu}=0.7$ in the critical phase. (b) The interacting case at the critical point.

numerically calculate the mutual information $I_{A B}=S_{A B}^{(2)}-$ $S_{A}^{(2)}-S_{B}^{(2)}$ between two intervals $A=\left[x_{1}, x_{2}\right]$ and $B=\left[x_{3}, x_{4}\right]$, where $x_{i}$ denotes the site, in the SYK chain with periodic boundary (see Fig. 4) and we observe that it is a function of cross ratio $\eta=$ $\left(\sin \frac{\pi}{L} x_{12} \sin \frac{\pi}{L} x_{34} / \sin \frac{\pi}{L} x_{14} \sin \frac{\pi}{L} x_{23}\right)$ with $x_{i j}=\left|x_{i}-x_{j}\right|$. In particular, we have $I_{A B} \sim \eta^{\Delta}$ in the limit $\eta \rightarrow 0$. In the critical phase of the free system, $\Delta \approx 1$ and at the critical point of the interacting system, $\Delta \approx 2$. We observe that these critical exponents are consistent with the previous numerical results in the small- $N$ case $[2,3,24]$.

Conclusions.-To summarize, we investigate the measurement-induced entanglement phase transition in Brownian SYK chains in the large- $N$ limit. We show that the dynamical symmetry in the replica space plays a crucial role: a $O(2)$ symmetry underlies the physics of noninteracting cases, and it is lowered to $C_{4}$ by finite interactions. The entanglement entropy in both cases can be understood in a unified framework by mapping to free energy costs of topological defects created by the twisted boundary conditions.

We acknowledge helpful discussions with Ehud Altman, Yimu Bao, Subhayan Sahu, and Greg Bentsen. S. K. J. and B. G. S. are supported by the Simons Foundation via the It From Qubit Collaboration. The work of B. G. S. is also supported in part by the AFOSR under Grant No. FA955019-1-0360. C. L. is supported by the NSF CMMT program under Grants No. DMR-1818533. P.Z. acknowledges support from the Walter Burke Institute for Theoretical Physics at Caltech. We acknowledge the University of Maryland High Performance Computing Cluster (HPCC).

\footnotetext{
*These authors contributed equally to this work.

†chenaad@bc.edu

tbswingle@umd.edu

\$pzhang93@caltech.edu

[1] Y. Li, X. Chen, and M. P. A. Fisher, Phys. Rev. B 98, 205136 (2018).
} 
[2] Y. Li, X. Chen, and M. P. A. Fisher, Phys. Rev. B 100, 134306 (2019).

[3] B. Skinner, J. Ruhman, and A. Nahum, Phys. Rev. X 9, 031009 (2019).

[4] M. J. Gullans and D. A. Huse, Phys. Rev. X 10, 041020 (2020).

[5] A. Chan, R. M. Nandkishore, M. Pretko, and G. Smith, Phys. Rev. B 99, 224307 (2019).

[6] A. Zabalo, M. J. Gullans, J. H. Wilson, S. Gopalakrishnan, D. A. Huse, and J. Pixley, Phys. Rev. B 101, 060301(R) (2020).

[7] M. J. Gullans and D. A. Huse, Phys. Rev. Lett. 125, 070606 (2020).

[8] Y. Li, X. Chen, A. W. Ludwig, and M. Fisher, arXiv: 2003.12721.

[9] R. Fan, S. Vijay, A. Vishwanath, and Y.-Z. You, Phys. Rev. B 103, 174309 (2021).

[10] J. Iaconis, A. Lucas, and X. Chen, Phys. Rev. B 102, 224311 (2020).

[11] S. Sang and T. H. Hsieh, Phys. Rev. Research 3, 023200 (2021).

[12] A. Lavasani, Y. Alavirad, and M. Barkeshli, Nat. Phys. 17, 342 (2021).

[13] M. Ippoliti, M. J. Gullans, S. Gopalakrishnan, D. A. Huse, and V. Khemani, Phys. Rev. X 11, 011030 (2021).

[14] G. Mazzucchi, W. Kozlowski, S. F. Caballero-Benitez, T. J. Elliott, and I. B. Mekhov, Phys. Rev. A 93, 023632 (2016).

[15] C. M. Bender and S. Boettcher, Phys. Rev. Lett. 80, 5243 (1998).

[16] Y. Ashida, S. Furukawa, and M. Ueda, Nat. Commun. 8, 15791 (2017).

[17] Y. Ashida, Z. Gong, and M. Ueda, Adv. Phys. 69, 249 (2020).

[18] A. Biella and M. Schiró, arXiv:2011.11620.

[19] S. Gopalakrishnan and M. J. Gullans, Phys. Rev. Lett. 126, 170503 (2021).

[20] S.-K. Jian, Z.-C. Yang, Z. Bi, and X. Chen, arXiv: 2101.04115.

[21] S. Choi, Y. Bao, X.-L. Qi, and E. Altman, Phys. Rev. Lett. 125, 030505 (2020).

[22] Y. Li and M. Fisher, Phys. Rev. B 103, 104306 (2021).

[23] J. Napp, R. L. La Placa, A. M. Dalzell, F. G. Brandao, and A. W. Harrow, arXiv:2001.00021.

[24] X. Chen, Y. Li, M. P. A. Fisher, and A. Lucas, Phys. Rev. Research 2, 033017 (2020).

[25] O. Alberton, M. Buchhold, and S. Diehl, Phys. Rev. Lett. 126, 170602 (2021).

[26] C.-M. Jian, B. Bauer, A. Keselman, and A. W. Ludwig, arXiv:2012.04666.

[27] Q. Tang, X. Chen, and W. Zhu, Phys. Rev. B 103, 174303 (2021).

[28] M. Buchhold, Y. Minoguchi, A. Altland, and S. Diehl, arXiv:2102.08381 [Phys. Rev. X (to be published)].

[29] Y. Bao, S. Choi, and E. Altman, arXiv:2102.09164.

[30] T. Zhou and A. Nahum, Phys. Rev. B 99, 174205 (2019).

[31] C.-M. Jian, Y.-Z. You, R. Vasseur, and A. W. W. Ludwig, Phys. Rev. B 101, 104302 (2020).

[32] Y. Bao, S. Choi, and E. Altman, Phys. Rev. B 101, 104301 (2020).
[33] P. Zhang, S.-K. Jian, C. Liu, and X. Chen, arXiv:2104.04088.

[34] A. Kitaev, Proceedings of the KITP Program: Entanglement in Strongly-Correlated Quantum Matter (Kavli Institute for Theoretical Physics, Santa Barbara, 2015), Vol. 7.

[35] S. Sachdev and J. Ye, Phys. Rev. Lett. 70, 3339 (1993).

[36] J. Maldacena and D. Stanford, Phys. Rev. D 94, 106002 (2016)

[37] P. Saad, S. H. Shenker, and D. Stanford, arXiv:1806.06840.

[38] C. Sünderhauf, L. Piroli, X.-L. Qi, N. Schuch, and J. I. Cirac, J. High Energy Phys. 11 (2019) 038.

[39] C. Liu, P. Zhang, and X. Chen, SciPost Phys. 10, 048 (2021).

[40] S.-K. Jian and B. Swingle, J. High Energy Phys. 03 (2021) 042.

[41] C. Liu, X. Chen, and L. Balents, Phys. Rev. B 97, 245126 (2018).

[42] Y. Gu, A. Lucas, and X.-L. Qi, J. High Energy Phys. 09 (2017) 120.

[43] Y. Huang and Y. Gu, Phys. Rev. D 100, 041901(R) (2019).

[44] P. Zhang and C. Liu, and X. Chen, SciPost Phys. 8, 094 (2020).

[45] A. Haldar, S. Bera, and S. Banerjee, Phys. Rev. Research 2, 033505 (2020).

[46] P. Zhang, J. High Energy Phys. 06 (2020) 143.

[47] Y. Chen, X.-L. Qi, and P. Zhang, J. High Energy Phys. 06 (2020) 121.

[48] A. M. García-García, Y. Jia, D. Rosa, and J. J. Verbaarschot, arXiv:2102.06630.

[49] X. Chen, R. Fan, Y. Chen, H. Zhai, and P. Zhang, Phys. Rev. Lett. 119, 207603 (2017).

[50] X.-Y. Song, C.-M. Jian, and L. Balents, Phys. Rev. Lett. 119, 216601 (2017).

[51] H. M. Wiseman and G. J. Milburn, Phys. Rev. Lett. 70, 548 (1993).

[52] H. J. Carmichael, Phys. Rev. Lett. 70, 2273 (1993).

[53] H. Wiseman, J. Eur. Opt. Soc. B 8, 205 (1996).

[54] C. Gardiner, P. Zoller, and P. Zoller, Quantum Noise: A Handbook of Markovian and Non-Markovian Quantum Stochastic Methods with Applications to Quantum Optics (Springer Science \& Business Media, New York, 2004).

[55] See Supplemental Material at http://link.aps.org/ supplemental/10.1103/PhysRevLett.127.140601 for (1) the effective action and the saddle-point equation for the monitored system; (2) the derivation of the Goldstone mode effective action; (3) the derivation of the $Z_{4}$ effective action; and (4) details of numerical calculations for Figs. 3 and 4.

[56] The symmetry is $[O(2) \times O(2)] \rtimes Z_{2}$. Since the $Z_{2}$ remains intact in the discussion, we simply neglect this semidirect product structure.

[57] Without the coupling between the left and the right chains, $\mu=0$, the action is invariant under two $O(2) \times O(2)$ for the left and the right chains, respectively, $G_{a b, x} \rightarrow O_{a}^{-1} G_{a b, x} O_{b}$, where $O_{a}^{T} O_{a}=1, O_{a}^{T} S O_{a}=1$.

[58] In this case we have $\delta G_{R R}^{12}=\delta G_{L L}^{12}, \quad \delta G_{R R}^{34}=\delta G_{L L}^{34}$, $\delta G_{R R}^{14}=\delta G_{L L}^{14}, \delta G_{R R}^{23}=\delta G_{L L}^{23}$ [55]. So we omit the subscript of the left and right chains.

[59] The trajectory-averaged Rényi entropy can be defined as $\tilde{S}_{A}^{(n)}=(1-n)^{-1} \mathbb{E} \operatorname{Tr} \rho \log \left(\operatorname{Tr} \rho_{A}^{n} / \operatorname{Tr} \rho^{n}\right)$. 
[60] G. Penington, S. H. Shenker, D. Stanford, and Z. Yang, arXiv:1911.11977.

[61] Starting from a maximally mixed density matrix, the quasientropy of the time evolved density matrix at time $T$ corresponds to imposing the twist boundary condition at $t=T$ boundary, and is given by $S^{(2)}=N \sigma L-\log (T / \sqrt{L})$, $T \gg L$ for periodic boundary condition for the chain. As expected, there are two phases corresponding to the volume-law and the area-law phases: when $\mu<J$, it takes an exponentially long time $\sqrt{L} e^{N \sigma L}$ to purify the state; when $\mu>J$, it takes a constant time $\sqrt{\xi}$ independent of system sizes to purify the state, where $\xi$ is the correlation length in the symmetric phase. The critical exponent is the same as the entanglement transition, as they differ only in boundary conditions. 